\title{
A NEW APPROACH FOR OPTICAL AND SAR SATELLITE IMAGE REGISTRATION
}

\author{
N. Merkle*, R. Müller, P. Schwind, G. Palubinskas, P. Reinartz
}

\author{
German Aerospace Center (DLR), Remote Sensing Technology Institute, Oberpfaffenhofen, 82234 Wessling, Germany \\ - (nina.merkle, rupert.mueller, peter.schwind, gintautas.palubinskas, peter.reinartz)@dlr.de
}

\author{
Commission I, WG I/4
}

KEY WORDS: Registration, Multisensor, SAR, Optical, Matching

\begin{abstract}
:
Over the last years several research studies have shown the high geometric accuracy of high resolution radar satellites like TerraSAR$X$. Due to this fact, the impact of high resolution SAR images for image registration has increased. An aim of high accuracy image registration is the improvement of the absolute geometric accuracy of optical images by using SAR images as references. High accuracy image registration is required for different remote sensing applications and is an on-going research topic. The registration of images acquired by different sensor types, like optical and SAR images, is a challenging task. In our work, a novel approach is proposed, which is a combination of the classical feature-based and intensity-based registration approaches. In the first step of the method, spatial features, here roundabouts, are detected in the optical image. In the second step, the detected features are used to generate SAR like roundabout templates. In the third step, the templates are matched with the corresponding parts of the SAR image by using an intensitybased matching process. The proposed method is tested for a pair of TerraSAR-X and QuickBird images and a pair of TerraSAR-X and WorldView-2 images of a suburban area. The results show that the proposed method offers an alternative approach compared to the common optical and SAR images registration methods and it can be used for the geometric accuracy improvement of optical images.
\end{abstract}

\section{INTRODUCTION}

Over the last years research studies, like Ager and Bresnahan (2009), have shown the high geometric accuracy of high resolution radar satellites like TerraSAR-X. In the papers of Reinartz et al. (2011) and Perko et al. (2011) methods are investigated which use high resolution TerraSAR-X images for the geometric accuracy improvement of optical images. The improvement of the geometric accuracy of the optical images is achieved by using ground control points (GCPs) selected from the SAR reference image. The methods used in Reinartz et al. (2011) and Perko et al. (2011) are based on multi-sensor image to image registration and use TerraSAR-X images as references. Image registration is required in different applications in remote sensing, like change detection or image fusion, and is an on-going research topic. The registration of images is a challenging task especially, if the images are acquired by different sensor types, like optical and SAR images. Commonly, image registration methods consist of four steps: (1) feature detection and extraction, (2) feature matching, (3) transformation model estimation and (4) image resampling and transformation (Zitov and Flusser, 2003). The proposed method described in this paper focuses only on the first two steps of the image registration. An overview of image registration techniques can be found in the papers of Brown (1992), Zitov and Flusser (2003) and Xiong and Zhang (2010).

Over the last years many different registration approaches were developed. The most common approaches for SAR and optical image registration can be classified into feature-based or intensitybased approaches. For the intensity-based registration approaches often mutual information is used (Chen et al. (2003), Suri and Reinartz (2010)). Wang and Tian (2011) proposed to combine mutual information with image gradient and Shu et al. (2005) with orientation information. A drawback of most of the intensitybased approaches is that reliable results are only achieved for image pairs with small misalignment (Zhao et al., 2014). Most

\footnotetext{
*Corresponding author. Email: nina.merkle@dlr.de.
}

of the feature-based approaches, like Cheng (2010) or Huang et al. (2010), are using point, line or area features for the registration. Other approaches using multi-feature for the image registration (Weijie et al. (2009), Wang et al. (2010)). A drawback of these approaches is the difficulties in the feature detection from the SAR image due to speckle noise and radar effects. The results of these registration approaches depend on the quality of the detected features from the SAR image. In this paper a new registration method is proposed which avoids the direct detection of features from the SAR image and combines the classical featurebased and intensity-based registration approaches. The outcome of the proposed method are GCPs selected from the SAR reference image. These GCPs can be used to compute the transformation parameters for the registration of the optical images to the SAR image. In cases where the geometric accuracy of the automatic georeferenced optical images is in the range of a few pixels, a transformation can be used for the registration improvement or for a direct improvement of the exterior orientation parameters or given rational polynomial coefficients (RPCs) (Müller et al., 2012). For the estimation of the affine transformation parameters only a few GCPs with a high geometric quality are required.

The proposed method consists of three main steps. The first step of the method is the detection of spatial features (here roundabouts) in the optical image, in the second step SAR like templates are generated from the detected features in the optical image and in the third step the templates are matched with the corresponding parts of the SAR image using an intensity-based matching method. Afterwards, the positions of the features in the SAR image are computed from the matching results. Promising results are obtained by applying the proposed method on two sets of optical and SAR image pairs. The results show that the proposed registration method is a new alternative to classical registration methods. It can be deduced from the results that the geometric accuracy of the optical image can be improved by using the SAR image as a reference. If the geometric accuracy of the SAR image is higher than of the optical image the achieved positions of the features in the SAR image can be used to generate transfor- 
mation parameters for the registration of the optical image to the SAR image.

In the following Section 2. the three main steps of the proposed method are described in detail. Section 3. gives an overview of the used test data. The obtained results are illustrated in Section 4. and discussed in Sections 5. The conclusion of the paper and the future work is stated in Section 6.

\section{METHOD}

The proposed method for the registration of optical and SAR images consists of: first, the generation of circle information (Section 2.1), second, the template generation using the circle information (Section 2.2) and third, the matching of the SAR image with the template (Section 2.3). Figure 1 illustrates an overview of the whole process, where the red marked path is only for validation purpose.

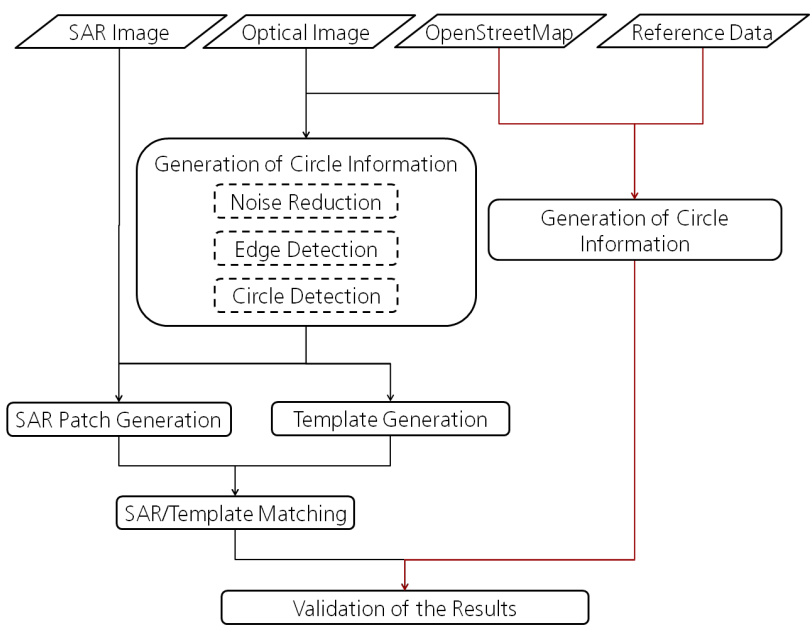

Figure 1. Overview of the proposed method. The red marked path is for validation purpose.

\subsection{Generation of Circle Information}

The first step of the template-based matching approach is the detection of spatial features in the optical images. In this paper the focus lies on the detection of roundabouts. A roundabout is a special type of road intersection and is composed of a circulatory roadway and a central island. Generally, the central island is bounded by a circle and contains vegetation like grass, plants or small trees.

The detection of roundabouts in the optical image starts with the detection of the central islands. Searching through the whole image for central islands is time consuming and often leads to a large number of false alarms. Therefore, OpenStreetMap (OSM) is used to get additional information about the roundabouts. In the whole area of Germany OSM provides information over more than 17000 roundabouts. For a chosen scene OSM provides the approximate position of the center $\left(\widetilde{x_{c i}}, \widetilde{y_{c i}}\right)$ and the approximate radius $\widetilde{R_{i}}$ of all central islands in the image, with $i=1, \ldots, N$ and $N$ is the number of roundabouts in the optical image. Since the accuracy of the GPS data, used for OSM, is 20 meters or better (OpenStreetMap Wiki (2014)), $\left(40 \mathrm{~m}+2 \widetilde{R_{i}}\right) \times\left(40 \mathrm{~m}+2 \widetilde{R_{i}}\right)$ search areas around the central island centers $\left(\widetilde{x_{c i}}, \widetilde{y_{c i}}\right)$ of each roundabout are defined. This information about the roundabouts, restricts the search area in the optical image, reduces the number of false detected circular objects, and increases the processing speed.

Since the detected edges of the image play a crucial role in the next steps, it is important to highlight the differences between the street and the vegetation around the street or inside the central island. Therefore, in the case of a multispectral image the normalized difference vegetation index (NDVI) is applied on the search areas. The NDVI of a multispectral image is calculated as follows

$$
\mathrm{NDVI}=\frac{\mathrm{NIR}-\mathrm{RED}}{\mathrm{NIR}+\mathrm{RED}}
$$

where NIR is the near infrared and RED is the red band (left image in Figure 2).

The next step, is to apply a method for the detection of circular features in each search area. The main concept of this method can be found in Wu et al. (2008), but to improve the accuracy some of the steps are changed.

First, a zero crossing edge detection method is applied on each search area. The search areas are filtered with the Laplacian of Gaussian (LoG) filter. The LoG filter is defined as follows

$$
\nabla^{2} G_{\sigma}(x, y)=\frac{1}{\pi \sigma^{4}}\left[1-\frac{x^{2}+y^{2}}{2 \sigma^{2}}\right] e^{-\frac{x^{2}+y^{2}}{2 \sigma^{2}}}
$$

where $\nabla^{2} f(x, y)=\frac{\partial^{2} f}{\partial^{2} x}+\frac{\partial^{2} f}{\partial^{2} y}$ is the Laplacian operator and $G_{\sigma}=\frac{1}{2 \pi \sigma^{2}} e^{-\frac{x^{2}+y^{2}}{2 \sigma^{2}}}$ is the Gaussian function. After filtering the image with the LoG function, the edges are found by looking for zero crossings. The right image in Figure 2 shows the result after applying the zero crossing edge detection method on the image shown on the left side.

In order to detect the circles in the image, the edge pixels which connect circular and non-circular objects have to be removed. This is done by using the gradient angle of each pixel. If an edge pixel $p$ lies on a circular object, then the gradient angle of $p$ differs only slightly from the gradient angle of adjacent edge pixels, which also lie on the same circular object. Therefore, all edge pixels whose gradient angle differs from the connected edge pixels mean gradient angel by a small threshold are removed. This step is more important for the detection of other circular objects than the central islands, because central islands are surrounded by streets and generally the edge pixels are separated from other objects.

In the next step, connected component labeling is used to divide the edge pixels into labeled components based on 8- neighborhood connectivity. Components with too few edge pixel (for example 5 edge pixels) have no influence on the circle detection and are removed in this step.

Furthermore, circles are fitted to the set of edge pixels of each component. The common problem of fitting circles to data can be solved by many different approaches. Gander et al. (1994) and Umbach and Jones (2003) compared several of these approaches with each other. The classical approach is the geometrical circle fit. The geometrical circle fit is based on orthogonal least squares and minimizes the function

$$
F\left(x_{c}, y_{c}, R\right)=\sum d_{i}^{2}
$$

where $d_{i}=r_{i}-R$ is the distance from the data point $\left(x_{i}, y_{i}\right)$ to the circle $(i=1, \ldots, M$ and $M$ is the number of data points), $r_{i}=\sqrt{\left(x_{i}-x_{c}\right)^{2}+\left(y_{i}-y_{c}\right)^{2}},\left(x_{c}, y_{c}\right)$ is the center and $R$ the 
radius of the circle. In this paper the data points are the edge pixels. Geometric based circle fit methods provide results with high accuracy, but these methods are all iterative and, hence, depending on a good initial guess. More information about the classical geometric circle fit can be found in Rusu et al. (2003).

Wu et al. (2008) use an algebraic circle fit method, which is often referred to as Kåsa fit. The algebraic circle fit methods are based on the algebraic circle equation

$$
a\left(x_{i}+y_{i}\right)^{2}+b x_{i}+c y_{i}+d=0
$$

with $a \neq 0$. Here, a circle is found by minimizing the function

$$
F(a, b, c, d)=\sum\left[a z_{i}+b x_{i}+c y_{i}+d\right]^{2}
$$

where $z_{i}=\left(x_{i}+y_{i}\right)^{2}$. The Kåsa fit is computationally fast, but the disadvantage is that the accuracy of the results depends on the arc length and on the noise in the data (Rusu et al., 2003). Thus, it is often used to get only a starting vector for a geometrical circle fit approach. The advantage of algebraic circle fits is that they have a closed form solution for the circle center and the radius and no iterations are needed.

In this paper another algebraic circle fit is used, named Hyper fit, which can be found in the paper of Al-Sharadqah and Chernov (2009). They investigated different algebraic circle fit methods and compared them with the classical geometrical approach. They reveal that the Hyper fit method, achieves even better results than the classical geometrical circle fit. The Hyper fit also minimizes the function from Eq. (4) but with the following constraint

$$
8 a^{2} \bar{z}+8 a b \bar{x}+8 c a \bar{y}+4 a d+b^{2}+c^{2}=1 .
$$

Using matrix representation this problem can be rewritten as follows

$$
\begin{array}{ll}
\text { minimize } & F(A)=A^{T} M A \\
\text { subject to } & A^{T} H A=1,
\end{array}
$$

where

$$
\begin{aligned}
A=(a, b, c, d)^{T}, M & =\frac{1}{n} Z^{T} Z, Z=\left(\begin{array}{cccc}
z_{1} & x_{1} & y_{1} & 1 \\
\vdots & \vdots & \vdots & \vdots \\
z_{n} & x_{n} & y_{n} & 1
\end{array}\right) \\
\text { and } H & =\left(\begin{array}{cccc}
8 \bar{z} & 4 \bar{x} & 4 \bar{y} & 2 \\
4 \bar{x} & 1 & 0 & 0 \\
4 \bar{y} & 0 & 1 & 0 \\
2 & 0 & 0 & 0
\end{array}\right) .
\end{aligned}
$$

Here, $\left(x_{i}, y_{i}\right)$ are the coordinates of the edge pixels from one component, $\bar{x}=\frac{1}{n} \sum x_{i}$ is the arithmetic mean and $n$ is the number of edge pixels in a component.

With the use of the Lagrange multipliers, the problem stated in Eq. (7) and (8) can be reduced to a generalized eigenvalue problem

$$
M A=\lambda H A \quad \text { subject to } \quad A^{T} H A=1 .
$$

Since, the overall problem is to minimize

$$
A^{T} M A=A^{T}(\lambda H A)=\lambda A^{T} H A=\lambda
$$

the solution of the generalized eigenvalue problem from equation (9) with the smallest $\lambda$ should be chosen ( $M=\frac{1}{n} Z^{T} Z$ is positive semi-definite and, hence, $\lambda$ is non-negative). Using a numerically stable procedure the solution of the generalized eigenvalue problem from equation (9) can be found. The circle center $\left(x_{c}, y_{c}\right)$ and the radius $R$ can be computed for each component. A more detailed explanation of the last mentioned step is described in (Al-Sharadqah and Chernov, 2009).

After using the Hyper fit method to fit a circle to the edge pixels of each component, valid circles have to be identified. A circle is valid if the edge pixels of a component lie on a circle or a circle arc. To identify components containing a set of edge pixels, which are lying on circles or circle arcs, Wu et al. (2008) described the following method. First, the geometric center

$$
\left(x_{m}, y_{m}\right)=\left(\frac{1}{n} \sum x_{i}, \frac{1}{n} \sum y_{i}\right)
$$

of each component is calculated from the corresponding set of edge pixels, where $\left(x_{i}, y_{i}\right)$ are the coordinates of the edge pixels and $n$ is the number of edge pixels in the component. Given a component, the corresponding set of edge pixels, the fitted circle center $\left(x_{c}, y_{c}\right)$ and the geometric center $\left(x_{m}, y_{m}\right)$. The line passing through $\left(x_{c}, y_{c}\right)$ and $\left(x_{m}, y_{m}\right)$ divides the set of edge pixels into two subsets. For both subsets a circle is fitted and the circle center and radius are calculated. If the distance between these circle centers and the difference of both radii are approximately equal, then the edge pixels of the component are lying on a circle. To prove this, the following formula is used

$$
\text { conf }=\left(1-\frac{\left\|R_{1}-R_{2}\right\|}{R_{1}+R_{2}}\right)\left(1-\frac{\left\|C_{1}-C_{2}\right\|}{R_{1}+R_{2}}\right)
$$

where $R_{1}$ and $R_{2}$ are the radii and $C_{1}$ and $C_{2}$ the circle centers of the two subsets. If the value conf of set of edge pixels is smaller than a threshold, then the edge pixels are not lying on a circle and the corresponding circle is removed.

Further circles are removed using additional information from OSM. Since OSM provides also the approximate radius of the central island, all circles whose radius has a large difference from the OSM central island radius are not further considered.

The last step is to reassemble some of the circles. In the edge pixel detection step a circle is split into different parts. Consequently, the edge pixels of this circle belong to different components. Hence, two circles are reassembled if the distance between their circle center positions and the difference between the radii are smaller than a threshold. Then, the corresponding components of these circles are jointed and a new circle is fitted to the jointed component. Figure 2 shows the detected circle after applying the above described steps superimposed on the NDVI image (left) and the detected edge image.
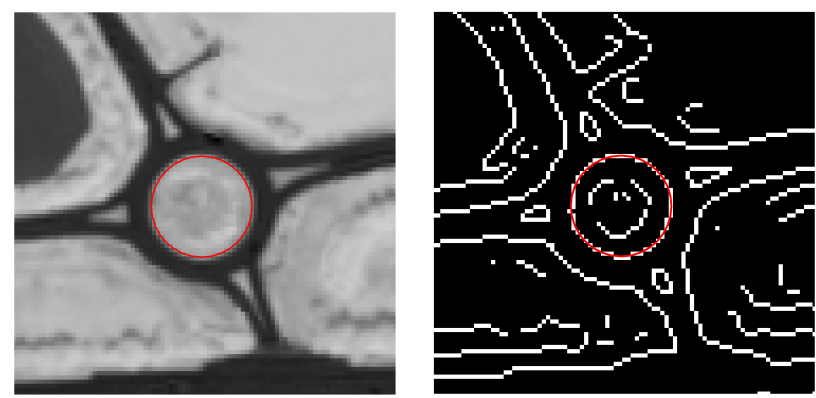

Figure 2. The detected circle (red) is superimposed on the NDVI image (left) and the detected edge image.

The obtaining results after all these steps are the centers $\left(x_{c i}, y_{c i}\right)$ and the radii $R_{i}$ of the central islands of the roundabouts, where 
$i=1, \ldots, N$ and $N$ the number of roundabouts in the optical image.

\subsection{Template Generation Using the Circle Information}

In this section the information about the roundabouts from Section 2.1 are used to generate roundabout templates. First, $\left(k_{i}+\right.$ $\left.2 R_{i}\right) \times\left(k_{i}+2 R_{i}\right)$ areas around the center of the central island $\left(x_{c i}, y_{c i}\right)$ are defined. The summand $k_{i}$ (in meter) depends on the size of the roundabout $i$.

The templates are generated from the above described areas of the optical images. The templates have the same size as the corresponding areas in the optical image and the center of the template is also the center of the corresponding central island. Each area contains a roundabout with a known center position and radius of the central island.

First, all pixels which belong to the street around the central island are detected. Therefore, a circle with radius $R_{i}+2 \mathrm{~m}$ around the center $\left(x_{c i}, y_{c i}\right)$ is defined (for an example see the top left image in Figure 3). The average width of the street around a central island is assumed to be $4 \mathrm{~m}$ or wider, thus the circle with radius $R_{i}+2 \mathrm{~m}$ is chosen. Then, the mean intensity value $\mu_{\text {street }}$ and the intensity variance $\sigma_{\text {street }}$ of all pixels, which lie on the circle are calculated. Since the circle lies on the street around the central island the mean intensity value $\mu_{\text {street }}$ should not vary much from the mean intensity value of the whole street. A pixel in the template is labeled to the street pixel class if the difference between the intensity value of the corresponding pixel in the optical image and $\mu_{\text {street }}$ is smaller than $\sigma_{\text {street }}$. The top right image in Figure 3 shows the street mask of the corresponding area in the optical image (top left). The street pixels are marked white and all other pixels are marked black.

All pixels, which belong to the boundary of the street area are labeled to the boundary pixel class. The non-labeled pixels, which are lying inside the circle with radius $R_{i}$ around the center $\left(x_{c i}, y_{c i}\right)$ are labeled to the central island pixel class. The bottom left image in Figure 3 shows the mask of the template (pixels belonging to the template are marked white).

Furthermore, an intensity value is assigned to each template pixel. The template pixels, which belong to the detected street are assigned with the intensity value $u_{\text {street }}$ and the template pixels, which belong to the central island are assigned with the intensity value $u_{\text {island }}$. The boundary pixels of the street and the central island are assigned with varying intensity values (described below).

Since the template is used for a matching process with the SAR image, roundabout templates are generated that have some similarities with the corresponding roundabouts in the SAR image. In SAR images there are different disturbing effects like shadows, layover, double bounces or speckle. For a more detailed explanation of these effects see (Stilla, 2007). In the roundabout templates only the double bounces are simulated, because effects like shadows and layover have more influence on above ground objects (The speckle noise is handled in the next section). The simulation of the double bounces does not consider the influence of the material on the reflections.

The double bounces occur at the curbstones, which are lying on the boundary of the central island or the surrounding street. The information from the SAR satellite, which are used to simulate the double bounce effects are the incidence angle (the angle between the radar beam and the surface normal) and the heading angle (rotation of azimuth heading clockwise w.r.t. North at midscene in degree (Fritz, 2007)). With these information the vector $v_{\text {sat }}$, which is pointing from the boundary pixel $p_{i}$ to the satellite is calculated. The other vector which is calculated is the gradient $v_{\operatorname{grad}_{i}}$ of the boundary pixel $p_{i}$.

Now, the angle $\alpha_{p_{i}}$ between the vectors $v_{\operatorname{grad}_{i}}$ and $v_{\text {sat }_{i}}$ at the boundary pixel $p_{i}$ is calculated. With the angle $\alpha_{p_{i}}$ the intensity of the boundary pixel $p_{i}$ is weighted and thereby, the double bounce effect is simulated. The template pixels belonging to the boundary are assigned with an intensity value of $w u_{\text {island, }}$, where $w=\frac{\alpha_{p_{i}}}{90^{\circ}}$ is the weighting factor for the pixel $p_{i}$. The result of the simulation can be seen in the bottom right image of Figure 3 .
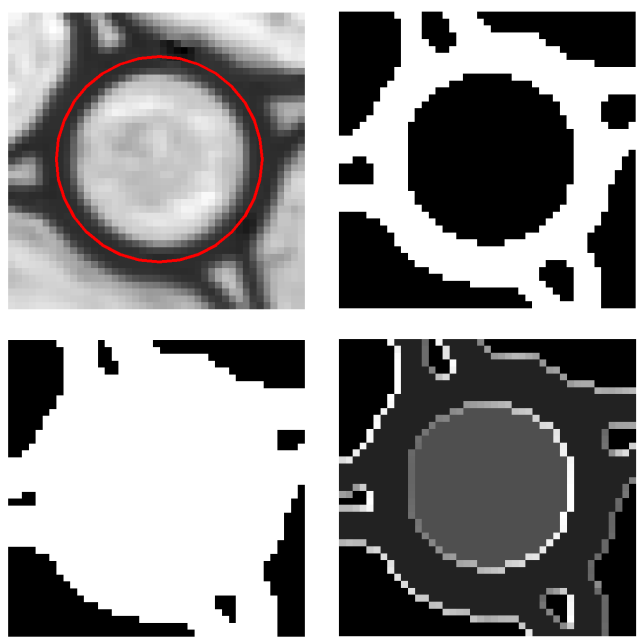

Figure 3. Illustration of the template generation. The roundabout in the optical image and the red marked circle inside the street (top left), the detected street (top right), the roundabout mask (bottom left) and the roundabout template (bottom right).

\subsection{SAR Image and Template Matching}

The last step of the proposed method is the SAR image and template matching. The aim of this section is to get the radius and the position of the centers of all central islands in the SAR image. Before the matching process, the roundabout templates and the SAR image are adapted to the same pixel size. The template is generated from the optical image and thus has the same pixel size as the optical image. To prevent the loss of information in the SAR image, all roundabout templates are resized to the pixel size of the SAR image by using bicubic interpolation.

Before computing the SAR image and template matching, the matching areas in the SAR image have to be restricted. For this purpose, SAR patches with a size of $\left(k R_{i}\right) \times\left(k R_{i}\right)$ around the central island centers $\left(x_{c i}, y_{c i}\right)$ are defined (see 2.1). The factor $k$ depends on the position accuracy of the chosen optical and SAR images. If the positions of the roundabouts in the SAR and the positions of the roundabouts in the optical image differs only by a few meters a smaller $k$ is used, otherwise $k$ must be chosen higher. Then, the adaptive Wiener filter (Lim, 1990) is applied to every SAR patch to reduce the speckle noise.

The template matching is done using normalized cross correlation (NCC). During the matching process the template is shifted by one pixel over the SAR image patch and for each position the NCC is calculated (see Figure 4). The templates have no regular shape, therefore the NCC of two vectors for the matching process 
is used. The NCC of two vectors is defined as follows

$$
\text { ncorr }=\frac{\sum_{i=1}^{N}\left(I_{i}-\bar{I}\right)\left(T_{i}-\bar{T}\right)}{\sqrt{\sum_{i=1}^{N}\left(I_{i}-\bar{I}\right)^{2}\left(T_{i}-\bar{T}\right)^{2}}},
$$

where $I_{i}$ and $T_{i}$ are the intensity values at position $i$ of the vectors $I$ and $T$, and $\bar{I}$ and $\bar{T}$ are the mean intensity values of the vectors $I$ and the $T$. The vector $T$ contains all intensity values of the template and the vector $I$ contains the intensity values of the SAR patch at the positions where the template and SAR patch are overlapping.

The position with the highest NCC value is the position where the similarity between the SAR patch and the template is highest and the match between the SAR image patch and the template is the best. Since the center of the template is also the center of the central island, the center of the central island in the SAR patch and, hence, in the SAR image can be calculated.
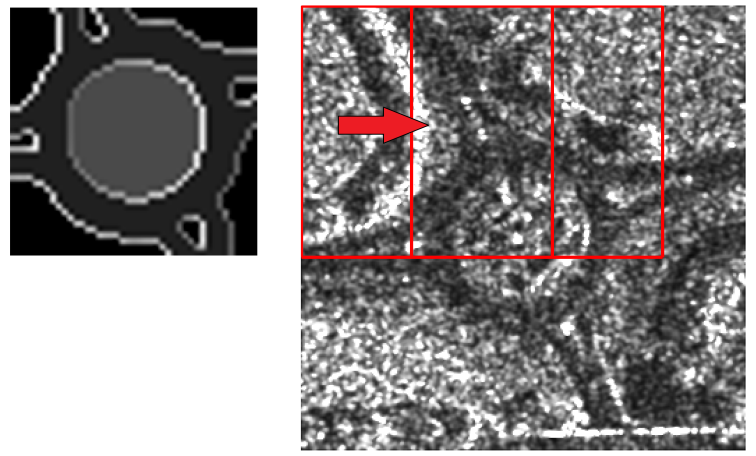

Figure 4. Illustration of the SAR image and template matching. The roundabout template (left) and the SAR image patch (right). The black areas around the template are no part of the template. The red marked areas in the SAR patch are two positions where the NCC is calculated.

\section{DATA}

The dataset includes TerraSAR-X, WorldView-2 and QuickBird imagery acquired over Oberpfaffenhofen, Germany (Table 1). The WorldView-2 and the QuickBird images are orthorectified using the Shuttle Radar Topography Mission (SRTM) DEM. The incidence angle of the TerraSAR-X satellite scene is $51.11^{\circ}$ and the heading angle is $188.14^{\circ}$. The spatial resolution of the TerraSAR$\mathrm{X}$ image is $0.5 \mathrm{~m}$, of the WorldView- 2 image $2 \mathrm{~m}$ and of the QuickBird image $2.4 \mathrm{~m}$. As reference data Digital Orthophotos (DOP) of the Federal Agency for Cartography and Geodesy with a pixel size of $20 \mathrm{~cm}$ and an absolute geometric accuracy of 60 $\mathrm{cm}$ are used. To cover all roundabouts in the TerraSAR-X and WorldView-2 scene, two QuickBird images and five Digital Orthophotos are used.

\section{RESULTS}

The proposed method described in Section 2. is tested for a pair of TerraSAR-X and QuickBird images and a pair of TerraSAR$\mathrm{X}$ and WorldView-2 images of a suburban area. There are five roundabouts in the scene with radii between $7.3 \mathrm{~m}$ and $24.9 \mathrm{~m}$. Using prior information provided by OSM (positions of the roundabouts and the radii of the central islands) four of the five roundabouts in the scene are detected. By the proposed method no objects are falsely detected as roundabouts.
For the detection of circles in the QuickBird, WorldView-2 and in reference images the same set of parameters are used. The parameters are tuned for the circle detection steps as follows: The window size of the LoG filter is $13 \times 13$ and $\sigma$ is 2 , components of the detected edge image with less than 10 pixels are removed, circles are valid if the corresponding value conf is less than 0.7 and, two circles are reassembled if their radii and the Euclidean distance between the circle centers differs by less than 1.5 pixel. Table 2 shows the radii of the roundabouts detected from the QuickBird, the WorldView-2 and in the reference images. With respect to the reference data the accuracy of the detected radii from the WorldView-2 is higher than of the detected radii from the QuickBird images.

The roundabout templates are generated as described in Section 2.2. For the areas around the central island centers the parameters $k_{i}$ are set as follows: $k_{1}=20 \mathrm{~m}, k_{2}=k_{5}=12 \mathrm{~m}$ and $k_{3}=8$ $\mathrm{m}$. The parameter $u_{\text {street }}$ is set to 35 and $u_{\text {island }}$ is set to 70 . With the described approach from Section 2.2 all roundabout templates are generated from the QuickBird and the WorldView-2 images without problems.

For the matching process described in Section 2.3 the parameter $k$ is set to 7 . The result after matching the roundabout template, which is generated from the first roundabout of the WorldView-2 image, with the TerraSAR-X image is shown in Figure 5. The image shows the NCC between the roundabout template and the corresponding TerraSAR-X image patch. A peak is at the position with the highest similarity between the template and the TerraSAR-X image. Here the value of the NCC is 0.2773 .

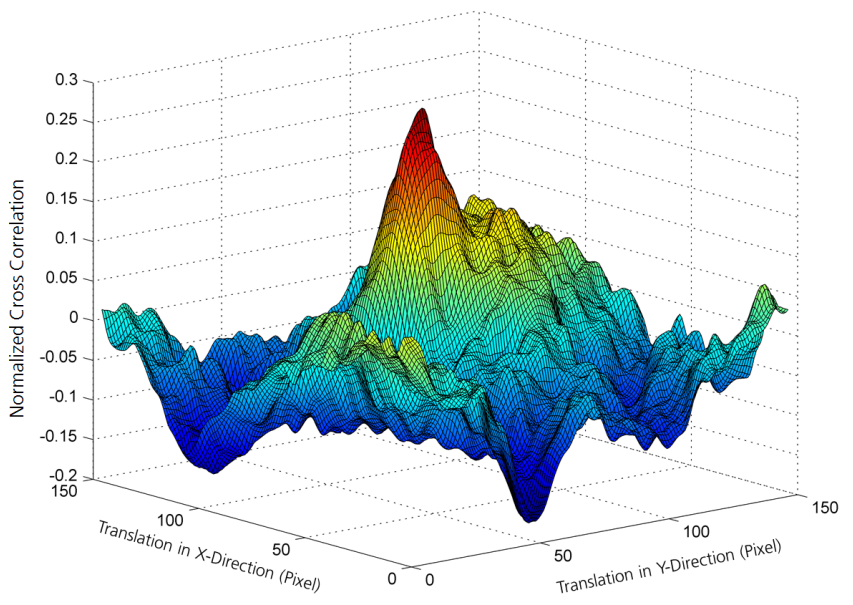

Figure 5. Normalized cross correlation between the TerraSAR-X image patch and the template generated from the first roundabout of the WorldView-2 image.

The left image in Figure 6 shows the roundabout template generated from the first roundabout of the WorldView-2 image. The right image of Figure 6 the TerraSAR-X image patch, which is the best match with the template.

After the matching process the positions of the central island center of each roundabout in the SAR image are calculated. Figure 7 shows a roundabout in the TerraSAR-X image and, the boundary and the center of the central island (red marked) achieved from the matching process.

To validate the results the roundabout centers and radii are detected in the reference data. The results in Table 3 are generated employing the QuickBird, the TerraSAR-X and the reference images. The results in Table 4 are generated employing 


\begin{tabular}{c||c|c|c|c} 
& TerraSAR- X & WorldView-2 & QuickBird & Reference \\
\hline \hline Mode & High resolution spotlight (HS) & Multispectral & Multispectral & RGB \\
\hline Pixel Size (m) & 0.5 & 2 & 2.4 & 0.2 \\
\hline Size (pixel) & $30000 \times 21000$ & $4774 \times 4420$ & $2408 \times 4233$ & $5000 \times 5000$ \\
\hline Processing Level & Enhanced Ellipsoid Corrected & $2 \mathrm{~A}$ & $2 \mathrm{~A}$ & Ortho \\
\hline Date of Acquisition & 19.07 .2014 & 22.8 .2011 & 24.9 .2011 and 24.10 .2011 & 25.6 .2012
\end{tabular}

Table 1. Details of TerraSAR-X, WorldView-2, QuickBird and the reference images.

\begin{tabular}{c||c|c|c} 
Roundabout & Radius from QuickBird $(\mathrm{m})$ & Radius from WorldView-2 $(\mathrm{m})$ & Radius from Reference $(\mathrm{m})$ \\
\hline \hline 1 & 23.67 & 23.77 & 24.86 \\
\hline 2 & 11.29 & 12.12 & 13.11 \\
\hline 3 & 6.86 & 6.60 & 7.32 \\
\hline 4 & - & - & - \\
\hline 5 & 9.87 & 9.98 & 10.91
\end{tabular}

Table 2. The roundabout radii detected from the QuickBird, the WorldView-2 and in the reference images.
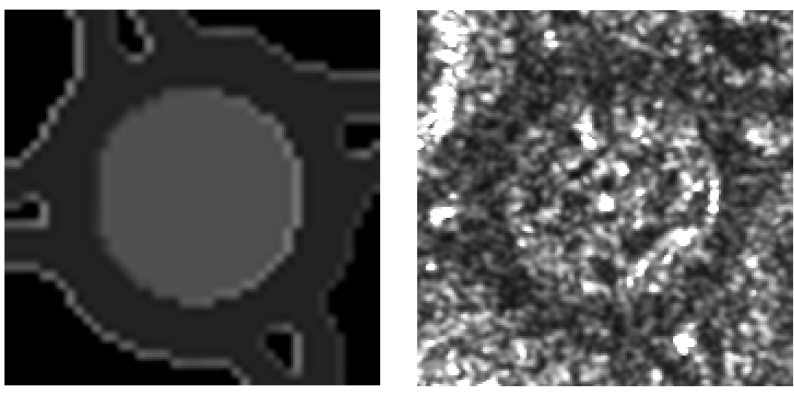

Figure 6. The roundabout template (left), which is generated from the first roundabout of the WorldView-2 image, and the corresponding area in the TerraSAR-X image with the highest NCC value (right). The corresponding NCC can be seen in Figure 5.

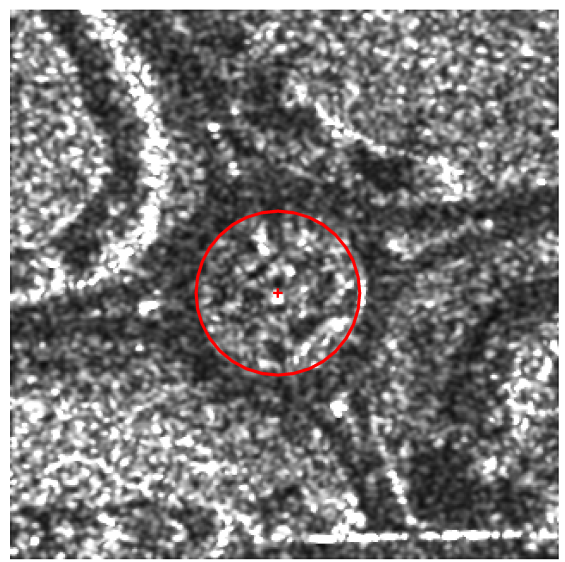

Figure 7. The SAR image and the resulting circle after the matching process.

the WorldView-2, TerraSAR-X and reference images. In both tables the distances $d_{x}$ and $d_{y}$ in $x$ - and $y$-direction, the Euclidean distances $d_{x, y}$, between the detected roundabout centers of the QuickBird and reference image, respectively the SAR and reference image are shown (the $x$-direction corresponds to east and the $y$-direction to north). Furthermore the mean deviation and the root mean square error (RMSE) are shown in this table.

The average distance between the detected roundabout centers of the QuickBird and the reference images is $6.88 \mathrm{~m}$ (Table 3). The differences in $x$-direction are higher than in $y$-direction. The average distance between the roundabout centers of the TerraSAR$\mathrm{X}$ and the reference images is $2.94 \mathrm{~m}$. The RMSE calculated from the distances between the center positions of the roundabouts in the TerraSAR-X and reference images is less than half of the RMSE calculated from the distances between the center positions of the roundabouts in the QuickBird and reference images.

The center positions of the roundabouts detected from the WorldView2 is in $x$-direction almost the same as the center positions of the roundabouts detected from the reference data (Table 4). In comparison to the QuickBird case the mean deviation and the RMSE have more than halved. The mean deviation and the RMSE calculated from the distances between the center positions of the roundabouts in the TerraSAR-X and reference images is slightly lower than the RMSE calculated from the distances between the center positions of the roundabouts in the WorldView-2 and reference images.

\section{DISCUSSION}

The results from Section 4. show that the proposed registration method is a promising alternative to the classical feature-based or intensity-based methods. In contrast to feature-based methods the features are only detected in the optical image and not in the SAR image. Based on the results the geometric accuracy of optical images can be improved by using the higher geometric accuracy of SAR images.

In the first step of the proposed method, the central islands of the roundabouts are detected in the Optical images. The detected radii from the QuickBird, the World-View-2 and from the reference images are shown in Table 2. The small differences between the radii detected from the WorldView-2 and QuickBird images can be explained by the different spatial resolutions of the images. The sizes of the detected radii of all three images differ roughly around $1 \mathrm{~m}$, although the pixel size of the WorldView-2 and QuickBird images is 10 times larger than the pixel size of the reference images. Accordingly, the proposed circle detection method achieves promising results with a high accuracy even for images with a pixel size around $2 \mathrm{~m}$. The fourth roundabout is not detected in the images. This is due to the fact that small trees are growing close to the boundary of the central island of roundabout four. These trees are overlaying some parts of the boundary. Consequently, no circle can be fitted to the corresponding set of edge pixels.

In the second step of the method, the roundabouts templates are generated from the optical images. The left image in Figure 6 shows the generated roundabout template from the first roundabout of the World-View-2 image. By comparing the template 


\begin{tabular}{c||c|c|c||c|c|c}
\multicolumn{1}{|c||}{ Roundabout } & \multicolumn{3}{c||}{ Distance between QuickBird and DOP } & \multicolumn{3}{c}{ Distance between SAR and DOP } \\
& $d_{x}[m]$ & $d_{y}[m]$ & $d_{x, y}[m]$ & $d_{x}[m]$ & $d_{y}[m]$ & $d_{x, y}[m]$ \\
\hline \hline 1 & -6.23 & 4.00 & 7.40 & 3.61 & -1.43 & 3.88 \\
\hline 2 & 4.8 & -3.25 & 5.79 & 1.23 & -0.53 & 1.34 \\
\hline 3 & -6.42 & 3.71 & 7.41 & -1.40 & 1.78 & 2.27 \\
\hline 4 & - & - & - & - & - & - \\
\hline 5 & -6.24 & 2.98 & 6.92 & 3.11 & -2.91 & 4.26 \\
\hline Mean Deviation & 5.92 & 3.49 & $\mathbf{6 . 8 8}$ & 2.34 & 1.66 & $\mathbf{2 . 9 4}$ \\
\hline RMSE & 5.96 & 3.51 & $\mathbf{6 . 9 1}$ & 2.56 & 1.87 & $\mathbf{3 . 1 7}$
\end{tabular}

Table 3. The distances $d_{x}$ and $d_{y}$ in $x$ - and $y$-direction, the Euclidean distances $d_{x, y}$, between the detected roundabout centers of the QuickBird and reference image, respectively the SAR and reference image, the mean deviation and, the root mean square error (RMSE). The $x$-direction corresponds to east and the $y$-direction to north.

\begin{tabular}{c||c|c|c||c|c|c}
\multirow{2}{*}{ Roundabout } & \multicolumn{2}{c||}{ Distance between WorldView-2 and DOP } & \multicolumn{3}{c}{ Distance between SAR and DOP } \\
& $d_{x}[m]$ & $d_{y}[m]$ & $d_{x, y}[m]$ & $d_{x}[m]$ & $d_{y}[m]$ & $d_{x, y}[m]$ \\
\hline \hline 1 & -0.17 & -2.90 & 2.91 & 1.48 & -0.42 & 1.54 \\
\hline 2 & -1.55 & -3.41 & 3.74 & 1.53 & -1.18 & 1.93 \\
\hline 3 & -0.68 & 3.31 & 3.37 & 3.57 & -2.06 & 4.12 \\
\hline 4 & - & - & - & - & - & - \\
\hline 5 & -0.11 & -2.87 & 2.87 & 2.77 & -2.10 & 3.48 \\
\hline Mean Deviation & 0.63 & 3.12 & $\mathbf{3 . 2 2}$ & 2.34 & 1.44 & $\mathbf{2 . 7 7}$ \\
\hline RMSE & 0.85 & 3.13 & $\mathbf{3 . 2 4}$ & 2.50 & 1.60 & $\mathbf{2 . 9 7}$
\end{tabular}

Table 4. The distances $d_{x}$ and $d_{y}$ in $x$ - and $y$-direction, the Euclidean distances $d_{x, y}$, between the detected roundabout centers of the WorldView-2 and reference image, respectively the SAR and reference image, the mean deviation and, the root mean square error (RMSE). The $x$-direction corresponds to east and the $y$-direction to north.

and the corresponding SAR image at the right side of Figure 6 the similarities between both can be seen. This example shows that the proposed template generation process is suitable for the generation of SAR like roundabout templates.

In the third step of the method, the roundabout templates are matched with the corresponding SAR image patch and the position of the roundabout in the SAR image is calculated. The results show (Figure 5 and 6 ) that by using NCC for the matching process, the position in the SAR image with the highest similarity to the template can be found. Since the template is shifted by one pixel over the SAR image only pixel accuracy can be achieved for the positions of the roundabouts. After the matching process the position of the roundabouts in the TerraSAR-X image are calculated. The example in Figure 7 shows that the accuracy of the achieved center positions and the radii of the roundabouts are quite high.

The achieved positions of the roundabout centers in the SAR image are twice as accurate as the positions of the roundabout centers in the QuickBird images (Table 3). To improve the geometric accuracy of the QuickBird images the TerraSAR-X image can be used. Therefore, the achieved center positions of the roundabouts of the TerraSAR-X image can be used to generate transformation parameters to map the QuickBird images to the TerraSAR-X image. With this method the geometric accuracy of the QuickBird image can be enhanced. But a limitation of this can be seen in Table 4, where the WorldView-2 image is used as the optical image. Here, the positions of the roundabouts centers detected from the WorldView- 2 are already very close to the reference data and the resulting positions of the roundabouts in the SAR images are only a bit more accurate. Therefore, in cases where the optical images have already a geometric accuracy around $3 \mathrm{~m}$ it is not of advantage to use the TerraSAR-X image and the proposed method to enhance the geometric accuracy further. In general, the proposed method can be used to improve the accuracy of optical images by using SAR images with a higher geometric accuracy than of the optical images.

\section{CONCLUSION AND FUTURE WORK}

A new method for the registration of optical and SAR images is proposed in this paper. The method consists of three main steps. The first step is the detection of the roundabout center positions and the radii of the central islands in the optical image. The second step is the generation of SAR like roundabout templates from the optical image. The third step is the matching process of the generated templates with the SAR image and the computation of the roundabout positions in the SAR image.

In contrast to common feature-based registration approaches, the features (here roundabouts) are only detected in the optical image. This is an advantage to the common feature-based registration approaches because the detection of spatial features in the SAR image is difficult due to the speckle noise and radar effects. The results show that in the case of a higher geometric accuracy in the SAR images than in the optical image the achieved center positions of the roundabouts from the SAR image can be used to improve the geometric accuracy of the optical image. Therefore, the center positions of the roundabouts can be used as GCPs to generate transformation parameters to improve the orientation of the optical image to the SAR image. For optical images from new very high resolution sensors like WorldView-2 or Pleiades, which already exhibit a geometric accuracy of a few meters a simple bias or an affine transformation of the RPCs is enough, which requires only a few GCPs with high geometric quality. Therefore, only a few features (like roundabouts) with high geometric accuracy have to be detected in the SAR image to improve the geometric accuracy of the optical image.

In future work the proposed method should be tested for further optical and SAR image pairs. Furthermore the generation of transformation parameters to improve the image orientation of the optical image should be added to the processing chain. Another interesting aspect for future work is the influence of the roundabout templates on the matching process and, hence, on the accuracy of the results. It will be investigated how important the size of the roundabouts and the simulation of the double bounces 
or other SAR effects are for the matching process and how the generation of the template could be further improved. To ensure that the method can also be used for images without roundabouts new features, like street junctions or highway exit ramps, should be included.

\section{ACKNOWLEDGEMENTS}

The authors would like to thank Janja Avbelj and Horst Hammer for their valuable suggestions and support.

\section{References}

Ager, T. and Bresnahan, P., 2009. Geometric precision in space radar imaging: results from TerraSAR-X. NGA CCAP Report.

Al-Sharadqah, A. and Chernov, N., 2009. Error analysis for circle fitting algorithms. Electronic Journal of Statistics Vol. 3, pp. 886-911.

Brown, L. G., 1992. A survey of image registration techniques. ACM Comput. Surv. 24(4), pp. 325-376.

Chen, H., Arora, M. K. and Varshney, P. K., 2003. Mutual information based image registration for remote sensing data. Int. J. Remote Sens 24, pp. 3701-3706.

Cheng, H., 2010. Optical image and SAR image matching based on scattered edge feature. In: 2010 International Conference on Multimedia Information Networking and Security (MINES), pp. 1-4.

Fritz, T., 2007. TerraSAR-X ground segment level $1 \mathrm{~b}$ product format specification. TX-GS-DD-3307 DLR Document.

Gander, W., Golub, G. H. and Strebel, R., 1994. Least-squares fitting of circles and ellipses. BIT Numerical Mathematics 34(4), pp. $558-578$

Huang, L., Li, Z. and Zhang, R., 2010. SAR and optical images registration using shape context. In: Geoscience and Remote Sensing Symposium (IGARSS), 2010 IEEE International, pp. 1007-1010.

Lim, J. S., 1990. Two-Dimensional Signal and Image Processing. Englewood Cliffs, Prentice Hall: NJ, pp. 536-540.

Müller, R., Krauß, T., Schneider, M. and Reinartz, P., 2012. Automated georeferencing of optical satellite data with integrated sensor model improvement. Photogrammetric Engineering \& Remote Sensing 78, pp. 61-74.

OpenStreetMap Wiki, 2014. Genauigkeit von GPS-Daten. http://wiki.openstreetmap.org/wiki/DE:Genauigkeit_von_GPSDaten, 19.11.2014.

Perko, R., Raggam, H., Gutjahr, K. and Schardt, M., 2011. Using worldwide available TerraSAR-x data to calibrate the geolocation accuracy of optical sensors. In: Geoscience and Remote Sensing Symposium (IGARSS), 2011 IEEE International, pp. 2551-2554.

Reinartz, P., Müller, R., Schwind, P., Suri, S. and Bamler, R., 2011. Orthorectification of VHR optical satellite data exploiting the geometric accuracy of TerraSAR-X data. ISPRS Journal of Photogrammetry and Remote Sensing 66, pp. 124-132.

Rusu, C., Tico, M., Kuosmanen, P. and Delp, E. J., 2003. Classical geometrical approach to circle fittingreview and new developments. Journal of Electronic Imaging 12(1), pp. 179-198.
Shu, L., Tan, T., Tang, M. and Pan, C., 2005. A novel registration method for SAR and SPOT images. In: IEEE International Conference on Image Processing, 2005. ICIP 2005, Vol. 2, pp. II-213-16.

Stilla, U., 2007. High resolution radar imaging of urban areas. In: Fritsch D. (ed) Photogrammetric Week, 07. Wichmann: Heidelberg pp. 149-158.

Suri, S. and Reinartz, P., 2010. Mutual-information-based registration of TerraSAR-X and IKONOS imagery in urban areas. IEEE Transactions on Geoscience and Remote Sensing 48(2), pp. 939-949.

Umbach, D. and Jones, K., 2003. A few methods for fitting circles to data. IEEE Transactions on Instrumentation and Measurement 52, pp. 1881-1885.

Wang, X. and Tian, J., 2011. Image registration based on maximization of gradient code mutual information. Image Analysis \& Stereology 24, pp. 1-7.

Wang, Z., Zhang, J., Zhang, Y. and Zou, B., 2010. Automatic registration of sar and optical image based on multi-features and multi-constraints. In: Geoscience and Remote Sensing Symposium (IGARSS), 2010 IEEE International, pp. 10191022.

Weijie, J., Jixian, Z. and Jinghui, Y., 2009. Automatic registration of SAR and optics image based on multi-features on suburban areas. In: Urban Remote Sensing Event, 2009 Joint, pp. 1-7.

Wu, J., Li, J., Xiao, C., Tan, F. and Caidong, G., 2008. Realtime robust algorithm for circle object detection. In: Young Computer Scientists, 2008. The 9th International Conference for, pp. 1722-1727.

Xiong, Z. and Zhang, Y., 2010. A critical review of image registration methods. International Journal of Image and Data Fusion 1(2), pp. 137-158.

Zhao, J., Gao, S., Sui, H., Li, Y. and Li, L., 2014. Automatic registration of SAR and optical image based on line and graph spectral theory. ISPRS - International Archives of the Photogrammetry, Remote Sensing and Spatial Information Sciences XL-4, pp. 377-382.

Zitov, B. and Flusser, J., 2003. Image registration methods: a survey. Image and Vision Computing 21, pp. 977-1000. 